\title{
A NEW ALGORITHM FOR CELL TRACKING TECHNIQUE
}

\author{
Ashraf A. Aly ${ }^{1}$, Safaai Bin Deris ${ }^{2}$ and Nazar Zaki ${ }^{3}$ \\ ${ }^{1,2}$ Faculty of Computer Science, Universiti Teknologi, Malaysia \\ Ashraf.ahmed@uaeu.ac.ae, safaai@utm.my \\ ${ }^{3}$ College of Information Technology, UAE University, UAE \\ nzaki@uaeu.ac.ae
}

\begin{abstract}
Tracking cell mobility analysis is essential process in many biology studies. Accurate and sensitive cell tracking system is essential to cell motility studies. To improve the overall living cells tracking systems performance and improve the accuracy, we focused on developing a novel algorithm for image processing. The algorithm we propose presents novel image segmentation and tracking system technique to incorporate the advantages of both Topological Alignments and snakes for more accurate tracking approach. First we apply the Topological Alignments and then transformed the output into the input of the active contour model to begin the analysis to the cells boundaries and determine the cells mobility. The results demonstrate that the proposed algorithm achieve accurate tracking for detecting and analyzing the mobility of the living cells. Our results indicate better segmentation and more accurate tracking for detecting and analyzing the mobility of the living cells, also the ability of the system to improve the low contrast, under and over segmentation.
\end{abstract}

\section{KEYWORDS}

Cell Tracking, Segmentation Enhancement, Active Contour, Topological Alignments, Image Segmentation, Mobility Analysis.

\section{INTRODUCTION}

Tracking cell mobility is an important part of many biological processes. Tissue cells of multi cellular organisms mobilize during embryologic development, generation of new blood vessels, cancer metastasis, and immune response. Understanding the mechanisms of cell motility is essential part for curative and preventative treatments to many diseases. Cell tracking and segmentation with high accuracy is important step in the cell motility research.

Segmentation is an essential part in many signal processing techniques and its applications. Texture analysis is important in many areas such as image processing, determination of the object shape, scene analysis. The process of segmentation depends on the determination of the best positions of the points which represent the image. 
The purpose of image segmentation is to partition an image into meaningful regions based on measurements taken from the image and might be grey level, colour, texture, depth or motion. Usually the process to determine the image starts with image segmentation as initial step. In this paper we introduce a new technique for image segmentation and cell tracking.

\section{RELATED WORK}

Cell mobility analysis is essential process in many biology studies. Tracking the number and velocity of rolling leukocytes is essential to understand and successfully treat inflammatory diseases as in Ray (2002). Sensitive tracking for moving cells is important to do mathematical modeling to cell locomotion. Coskun (2007) used imaging data to solve the inverse modeling problem to determine the mobility analysis of the cells. Li (2006) used a technique with two stages; the first one is a tracker and a filter to detect the cell and also the cells which move in and out of the image area. Another research by Mukherjee (2004), he developed a technique to handle the segmentation process and the tracking problem simultaneously. Moreover, Zimmer (2002) modified the snake model to track the movement of the cells and segment the first frame. Recently, a number of researchers have been created automated techniques to track and detect the cells mobility.

\section{ALGORITHM}

Topological alignments and snakes are used in image processing, especially in locating object boundaries. Each method has its own advantages and also limitations. Active contour (snakes), can locate the object boundaries dynamically and automatically from an initial contour. The advantage of Snakes model is the ability of the model to give a linear determination of the object shape at the convergence time, and no extra processing is needed. But Snakes model require detecting strong image gradients to detect the contour. This actually limits the use of Snakes, because weak boundaries of the image frames and also frames with low contrast will cause over and under segmentation which responsible for decreasing the accuracy of the analysis. To mitigate the effect of this problem with the Snakes model, to improve the performance of segmentation and cell tracking, we apply the Topological Alignments method to increase the accuracy of cell tracking and detecting analysis. The Topological Alignments method links segments between every frame and the next one; that will decrease the number of false detections and also the false trajectories.

In this paper, we introduce a novel technique based on active contour in conjunction with Topological Alignments. We present tracking system and image segmentation algorithm to incorporate the advantages of both active contour and Topological Alignments to get a tracking system with high accuracy to detect and analyze the mobility of the living cells. The novel technique proceeds in two steps: First we apply the Topological Alignments and then transformed the output into the input of the active contour model to begin the analysis to the cells boundaries and determine the cells mobility..

We have validated the novel technique by using an image protocol from Rodrigo (2007). We used tests on 70 images of human blood cells. The novel image processing technique is based on Open CV library (Bradski 2000). We implemented the algorithm in C++ under Windows XP operating system.

\subsection{Topological Alignments Method}

The topological alignments method based on linking the segmentation of two frames in the video sequence. Miura et al. [9]; Danuser et al.[17]; Zimmer et al.[8]; and Ersoy et al.[12]. From 
the output of the segmentation procedure, the method found the maximum weighted solutions between two pairs of frames. Then match the segments.

The topological alignments method represent the segmentation of two images from the video sequence as $m$ and $n$, index set $P=\{1, \ldots, m\}$, and an index set $Q=\{1, \ldots, n\}$.

The method assume that cells move moderately between two consecutive frames, we assign the relative overlap of $p$ and $q$ as their weight, formally defined as

$w(p, q):=|A(p) \cup A(q)| /|A(p) \cup A(q)|$

We determine the notation of the topological alignments based on these weights. We denote $P L(M)$ for the set of all $L$-partitioning`s of a finite set $M$, and $S$ as a family of sets and to identify the $L$ subsets as $S=(S 1, \ldots, S \mathrm{~L})$.

The method improves the cell tracking performance by improving the segmentation. The topological alignments method links segments between every frame and the next one; that will decrease the number of false detections and also the false trajectories.

\subsection{Active Contour Models}

Active contour (Snakes) method depends on segmenting an image using the deformation of the initial contour towards the object of interest boundaries. The method deforming the initial contour and minimizing the energy function for the contours, as in Kass et al. [3]; Ray et al. [6]; Zimmer et al. [7] and Sacan et al. [16]. We have here two components which represents the energy function; the first part is the potential energy component, and the potential energy component is small when the contour is aligned to the image edge, and the second part is the internal deformation energy component, and the component is small when the contour is smooth. The termination functional can be implemented with a gradient direction calculus in a slightly smoothed version of the image.

An Active contour can be parametrically represented by $\mathrm{v}(s)=(x(s), y(\mathrm{~s}))$ and its energy functional can be written as:

$E=\int_{0}^{1} E_{\text {int }}(v(s)) d s+\int_{0}^{1} E_{\text {image }}(v(s)) d s+\int_{0}^{1} E_{\text {ext }}(v(s)) d s$

Where $E_{\text {int }}$ represents the internal energy of the spline due to bending, $E_{\text {image }}$ gives rise to the image forces and $E_{\text {ext }}$ gives rise to the external constraint forces. However $a(s)$ and $B(s)$ will control the spline energy. Therefore, the internal spline energy can be written as:

$E_{\text {int }}=\frac{\left(a(s)\left|v_{s}(s)\right|^{2}+B(s)\left|v_{s S}(s)\right|^{2}\right)}{2}$

We represent the generic total image energy as a combination of the three weighted energy functions.

$$
E_{\text {image }}=w_{\text {line }} E_{\text {line }}+w_{\text {edge }} E_{\text {edge }}+w_{\text {term }} E_{\text {term }}
$$

Snakes can be represented by two models depends on the characteristics of the image; edgebased models and region based models. The advantage of Snakes model is the ability of the model to give a linear determination of the object shape at the convergence time, and no extra 
processing is needed. But Snakes model require detecting strong image gradients to detect the contour.

This actually limits the use of Snakes, because weak boundaries of the image frames and also frames with low contrast will cause over and under segmentation which responsible for decreasing the accuracy of the analysis.

\section{RESULTS AND DISCUSSION}

In order to obtain validation of our approach, we have tested the algorithms in by using two different protocols, live cells video sequences were collected and stored in database, and we are going to make comparison between automatic tracking and human controlled tracking. We got images for 40 living cells to be used to test and evaluate our tracking system. The other protocol contains a set of video sequences for leukocytes cells previously used in Jung (1998), (cmgm.stanford).

The automated tracker was used to compute the corresponding 40 cell positions and the cell shape change. Manual tracking measurements were obtained by allowing an operator to observe the cells movement, its movements were tracked on the computer monitor with the help of a tracking system as function of time and position. The cell velocity is calculated from the mass center across the frames. Some results for both manual and automated tracking of the leukocytes are given in Table 1.

Average movement velocity was $v=4.2 \pm 0.4 \mu \mathrm{m} / \mathrm{min}$ (manual) and $4.8 \pm 0.5 \mu \mathrm{m} / \mathrm{min}$ (automatic), consistent with earlier observations of Jung (1998). The RMSE between the manual and the computed displacement was less than $12 \%$ on average. The active contour method gave a velocity and change shape RMSE of less than $11 \%$, improves to less than $7 \%$ by using the novel algorithm presented here. The results indicates better mobility analysis for speed and changing shape due to the improvement in segmentation performance by using the topological Alignments, which leads to improve cell tracking results.

Our results indicate better segmentation and more accurate tracking for detecting and analyzing the mobility of the living cells. We have achieved better tracking and detecting the cells, also the ability of the system to improve the low contrast, under and over segmentation as shown in Figure 1, Figure 2 and Figure 3. Figure 4 and Figure 5 show the novel velocity and the shape index for the cells. The novel image processing technique used in the tracking system successfully address the major problems associated with tracking cells, image with low contrast, under and over segmentation; more accurate tracking for detecting and analyzing the mobility of the living cells.

\section{CONCLUSION}

Tracking cell mobility is an essential part of many biological processes. Topological alignments and snakes are used in image processing, especially in locating object boundaries. Each method has its own advantages and also limitations.. Active contour, can locate the object boundaries dynamically and automatically from an initial contour. The advantage of Snakes model is the ability of the model to give a linear determination of the object shape at the convergence time, and no extra processing is needed. But Snakes model require detecting strong image gradients to detect the contour. This actually limits the use of Snakes, because weak boundaries of the image frames and also frames with low contrast will cause over and under segmentation which responsible for decreasing the accuracy of the analysis. To mitigate the effect of this problem with the Snakes model, to improve the performance of segmentation and cell tracking, we apply 
the Topological Alignments method to increase the accuracy of cell tracking and detecting analysis.

In our experiments, we made comparison between our algorithm and traditional snake. The results show that the algorithm can demonstrate the segmentation accuracy under weak image boundaries, low contrast, under and over segmentation of living cells, which the most cell tracking challenge problems and responsible for lacking accuracy in cell tracking techniques.

The results show more accurate tracking for detecting and analyzing the mobility of living cells. Our results indicate better segmentation and more accurate tracking for detecting and analyzing the mobility of the living cells. We have achieved better tracking and detecting for living cells, also the ability of the system to enhance the segmentation for low contrast, under and over segmentation problem. In this paper we focused on solving the under and over segmentation and low contrast problems, however in our future work we will consider other problems with image segmentation such as images with high noise.

Table 1. Some mobility results for both manual and automated tracking of the leukocytes cells.

\begin{tabular}{|c|c|c|c|c|c|}
\hline Cell & $V e l_{M}(\mu \mathrm{m} / \mathrm{min})$ & $V e l_{U N}(\mu \mathrm{m} / \mathrm{min})$ & $V e l_{U N}(\mu \mathrm{m} / \mathrm{min})$ & Shape.Index $_{U}$ & Shape.index $_{U N}$ \\
\hline 1 & 7.0 & 7.3 & 7.4 & 4.4 & 4.5 \\
\hline 2 & 3.5 & 3.4 & 3.5 & 2.4 & 2.5 \\
\hline 3 & 2.5 & 2.6 & 2.8 & 1.5 & 1.6 \\
\hline 4 & 2.1 & 2.3 & 2.4 & 2.2 & 2.4 \\
\hline 5 & 3.1 & 4.3 & 4.1 & 2.5 & 2.5 \\
\hline 6 & 5.6 & 5.8 & 6.4 & 1.5 & 1.7 \\
\hline 7 & 6.8 & 6.7 & 6.8 & 4.3 & 4.6 \\
\hline 8 & 7.5 & 7.4 & 6.5 & 4.5 & 4.8 \\
\hline 9 & 2.3 & 2.2 & 2.6 & 3.1 & 3.1 \\
\hline 10 & 2.4 & 2.6 & 2.9 & 1.7 & 1.7 \\
\hline 11 & 3.5 & 4.3 & 4.9 & 2.5 & 2.4 \\
\hline 12 & 7.6 & 7.8 & 7.6 & 2.6 & 2.5 \\
\hline 13 & 2.8 & 3.2 & 3.2 & 2.8 & 2.9 \\
\hline 14 & 2.6 & 3.3 & 3.6 & 2.1 & 2.7 \\
\hline 15 & 2.4 & 3.3 & 3.6 & 2.1 & 2.7 \\
\hline 16 & 7.5 & 7.7 & 7.7 & 2.6 & 2.8 \\
\hline 17 & 4.3 & 4.8 & 5.1 & 2.3 & 2.6 \\
\hline 18 & 4.5 & 4.7 & 4.9 & 1.3 & 1.5 \\
\hline 19 & 7.8 & 7.6 & 7.7 & 2.5 & 2.6 \\
\hline 20 & 1.6 & 1.8 & 1.9 & 1.1 & 1.5 \\
\hline
\end{tabular}

$\operatorname{Vel}_{M}:$ MANUAL VELOCITY $\quad \operatorname{Vel}_{U}:$ AUTOMATED VELOCITY $\quad \operatorname{Vel}_{\text {UN }}:$ AUTO. (NOVEL) VELOCITY

Shape.Index $_{U}$ : CHANGE SHAPE INDEX Shape.index ${ }_{U N}$ :CHANGE SHAPE INDEX (NOVEL) 

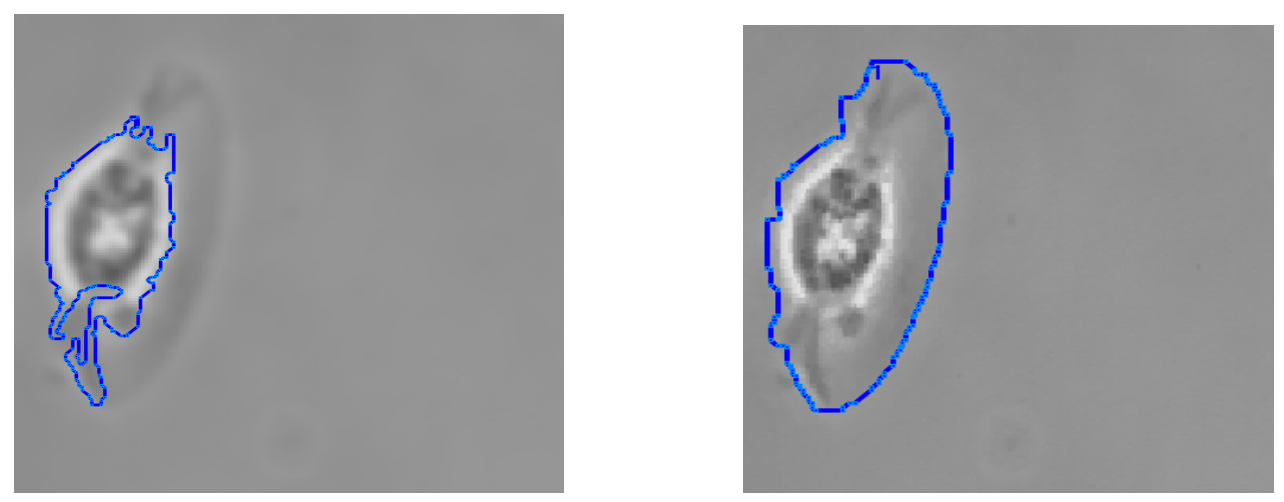

Fig. 1. Example images from image sequence.(a) Original capture Segmentation by using Active contour (b) Better contrast and segmentation by using the Novel algorithm by using the Novel algorithm.
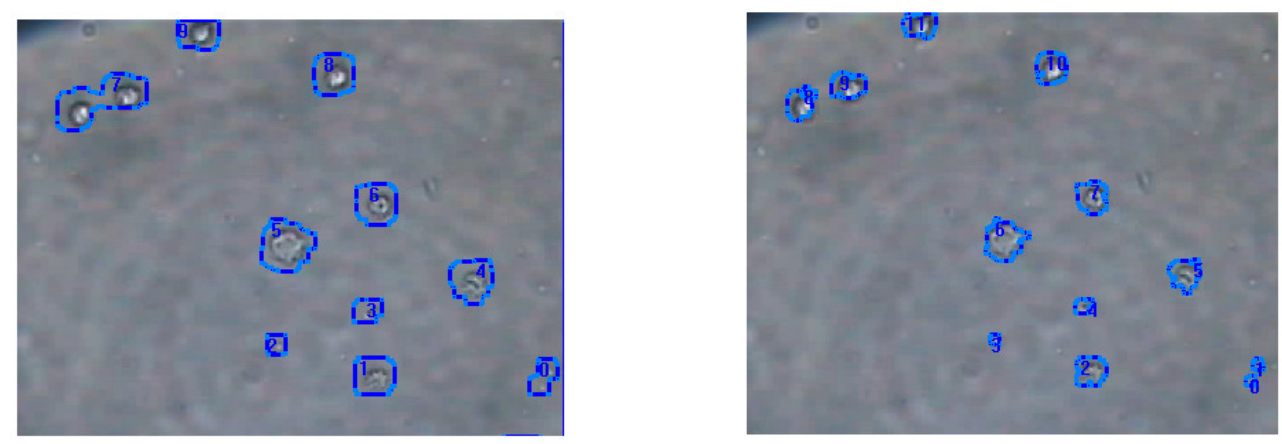

Fig. 2. Example images from image sequence (a) Under segmentation problem, (b) Under segmentation solved by using Active contour by using the Novel algorithm.
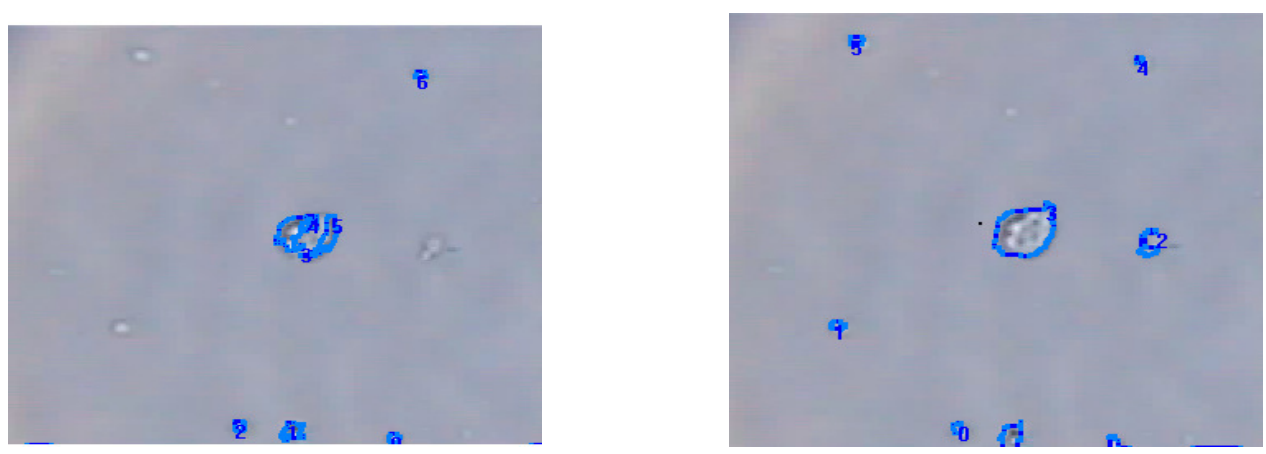

Fig. 3. Example images from image sequence (a) Over segmentation problem by using Active contour (b) Over segmentation problem solved, by using Active contour by using the Novel algorithm. 


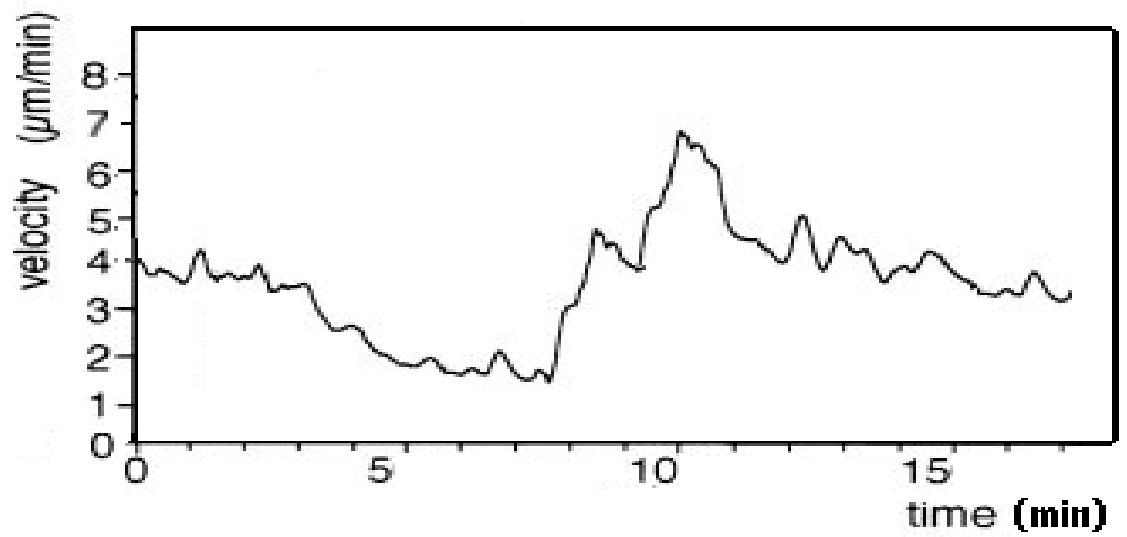

Fig. 4. Cells Velocity $\left(\operatorname{Vel}_{U N}\right)$

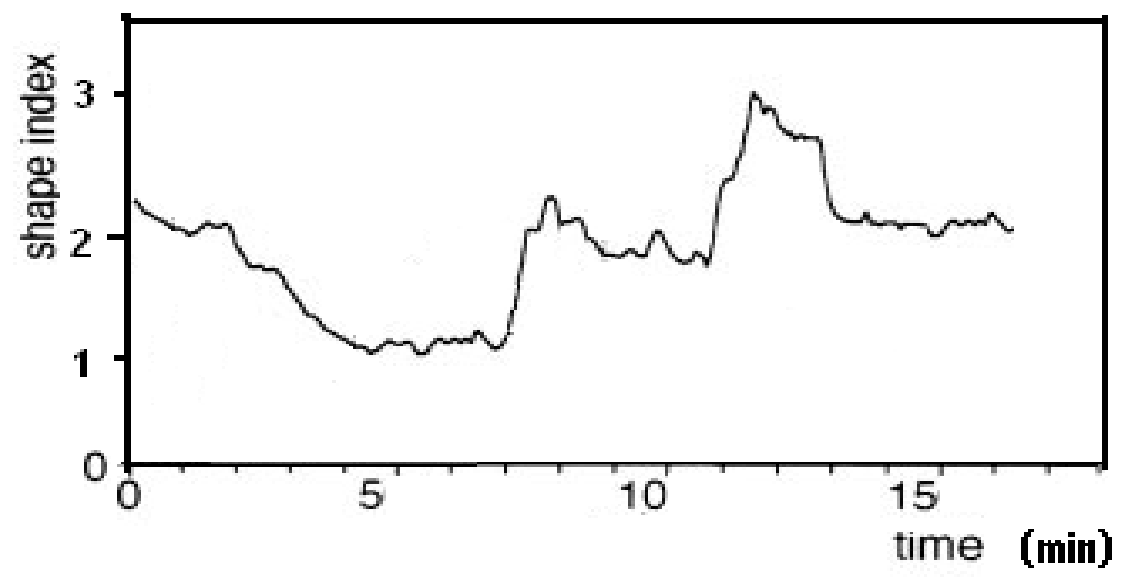

Fig. 5. Shape.index $x_{U N}$

\section{REFERENCES}

[1] Melange, T., Nachtegael, M. \& Kerre, E. E. (2011). Fuzzy Random Impulse Noise Removal From Color Image Sequences. IEEE Trans. 20: 1023 - 1035.

[2] Danuser, G., Meijering, E., and Smal, I. (2011).Tracking in molecular bioimaging. Signal Processing Magazine, IEEE, 23(3):46-53.

[3] Melange, T., Nachtegael, M. \& Kerre, E. E. (2011). Fuzzy Random Impulse Noise Removal From Color Image Sequences. IEEE Trans. 20: 1023 - 1035.

[4] Danuser, G., Meijering, E., and Smal, I. (2011). Tracking in molecular bioimaging. Signal Processing Magazine, IEEE, 23(3):46-53.

[5] Mignotte, M. (2010). A Label Field Fusion Bayesian Model and Its Penalized Maximum Rand Estimator for Image Segmentation. IEEE Trans. 19: 1610 - 1624.

[6] Mignotte, M. (2010). A Label Field Fusion Bayesian Model and Its Penalized Maximum Rand Estimator for Image Segmentation. IEEE Trans. 19: 1610 - 1624. 
[7] Palaniappan, K., Ersoy, I., Nath, S. K. (2007). Moving Object Segmentation Using the Flux Tensor for Biological Video Microscopy. Lect Notes Comput Sci. 4810: 483-493.

[8] Li, K., Miller, E., Weiss, L., Campbell, P., and Kanade, T. (2006). Online tracking of migrating and proliferating cells imaged with phase-contrast microscopy. Proc. of the 2006 Conf,on Computer Vision and Pattern Recognition Workshop(CVPRW'06), pages 6572.

[9] Smart, J., Hock, K., and Csomor, S. (2005). Cross - Platform GUI Programming with wx Widgets. Prentice Hall PTR.

[10] Bradski, G. (2000). The Open CV Library. Dr. Dobb's Software Tools for the Professional Programmer.

[11] Coskun, H., Li, Y. \& Mackey, M. A. (2007) “Ameboid cell motility: A model and inverse problem, with an application to live cell imaging data", Journal of Theoretical Biology, 244(2):169-179.

[12] Kass, M., Witkin, A. \& Terzopoulos, D. (1987) "Snakes : Active contour models", International Journal of Computer Vision, pages 321-331.

[13] Li, K., Miller, E., Weiss, L., Campbell, P., \& Kanade, T. (2006) “Online tracking of migrating and proliferating cells imaged with phase-contrast microscopy" Proc. of the 2006 Conf, on Computer Vision and Pattern Recognition Workshop (CVPRW'06), pages 65-72.

[14] Mukherjee, D., Ray, N. \& Acton, S. (2004) "Level set analysis for leukocyte detection and tracking" IEEE Trans Image Process, 13(4):562-72.

[15] Ray, N., Acton, S. \& Ley, K. (2002) "Tracking leukocytes in vivo with shape and size constrained active contours", 21(10):1222-1235.

[16] Zimmer, C., Labruyre, E., Meas - Yedid, V., Guilln, N. \& Olivo - Marin, J. (2002) "Segmentation and tracking of migrating cells in videomicroscopy with parametric active contours: a tool for cell-based drug testing", IEEE Trans Med Imaging, 21(10):1212-21.

[17] Zimmer C., Zhang B., Dufour A., Thebaud A., Berlemont S., Meas-Yedid V. \& O Marin JC (2006) "On the Digital Trail of Mobile Cells", Signal Processing Magazine, 23(3):54-62.

[18] Miura K. ( 2004) "Tracking Movement in Cell Biology", Advances in Biochemical Engineering/Biotechnology, 95:267-295.

[19] Mukherjee D., Ray N. \& Acton S (2004) "Level set analysis for leukocyte detection and tracking", IEEE Trans Image Process, 13(4):562-572.

[20] Li Y., Zheng Y., Doermann D. \& Jaeger S (2008) "Script - Independent Text Line Segmentation in Freestyle Handwritten Documents", IEEE Trans Pattern Anal Mach Intell, 30(8):1313- 1329.

[21] Ersoy I., Bunyak F., Mackey M. \& Palaniappan K (2008) "Cell Segmentation Using Hessian Based Detection and Contour Evolution with Directional Derivatives", International Conference on Image Processing, 1804-1807.

[22] Jung, U., Norman, K. E., Ramos, C. L., Scharffetter - Kochanek, K., Beaudet, A. L. \& Ley, K. (1998) "Transit time of leukocytes rolling through venules controls cytokine-induced inflammatory cell recruitment in vivo", J. Clin. Invest. 102, 1526-1533.

[23] Ray N., Acton S. \& Ley K (2002) “Tracking leukocytes in vivo with shape and size constrained active contours", IEEE Trans Med Imaging, 21(10):1222-1235.

[24] Debeir O., Ham P., Kiss R. \& Decaestecker C. (2005) "Tracking of migrating cells under phase- contrast video microscopy with combined mean-shift processes", IEEE Trans Med Imaging, 24(6):697-711.

[25] Sacan A., Ferhatosmanoglu H. \& Coskun H. (2008) "CellTrack an opensource software for cell tracking and motility analysis", Bioinformatics, 24(14):1647-1649.

[26] Danuser G., Meijering E. \& Smal I. (2011) “Tracking in molecular bioimaging”, Signal Processing Magazine, IEEE, 23(3):46-53. 